\title{
Density Dependent Selection in Parthenogenetic and Self-Mating Populations*
}

\author{
Alan R. Templeton \\ Department of Human Genetics and the Society of Fellows, \\ University of Michigan, Ann Arbor, Michigan $48104^{\dagger}$
}

Received May 2, 1972

\begin{abstract}
The consequences of density dependent selection on genetically heterogeneous, diploid populations reproducing by self-mating or various parthenogenetic mechanisms is investigated. A logistic fitness function that depends upon both the genotype of an individual and the density of the population is used. Such a fitness function simultaneously determines the population size and the genotype frequencies. The equilibrium solutions to a one locus and two locus model are given as well as some generalizations to $n$ loci and nonlogistic fitness functions. Conditions are found that maintain several different genotypes simultaneously in the equilibrium population. The interaction of such selection with the genetic mechanisms which determine mode of reproduction in parthenogenetic populations is also discussed.
\end{abstract}

\section{INTRODUCTION}

A common model of population growth is one in which the size of a genetically homogeneous population is regulated by density dependent factors. A common evolutionary model is one in which constant fitness values are assigned to the genotypes found in the population and population growth is ignored. However, it seems plausible that the forces which regulate population growth could also affect differentially the various genotypes found in the population and thus influence the genetic evolution of the population. The assumptions of genetic homogeneity and constant fitness are both unrealistic in such a case, and it is therefore of considerable interest to examine the consequences of natural selection in genetically heterogeneous populations regulated by density dependent factors.

Roughgarden (1971), Charlesworth (1971) and Clarke (1972) have recently

* This work was carricd out in part while the author was a recipient of an NSF Predoctoral Fellowship.

† Present address: Department of Genetics, University of Hawaii, Biomed. Bldg. RmA-106, 1960 East West Road, Honolulu, Hawaii 96822. 
studied this problem in monoecious diploid populations with respect to a single locus. However, sexual hermaphroditic populations are not alone in having genetic heterogeneity since many parthenogenetic populations can also maintain a large amount of genetic diversity (White, 1970). The impact of constant selective forces on certain types of parthenogenetic populations has already been examined by Asher (1970, 1972) and Templeton and Rothman (1973). Their work shows that constant selective forces can maintain genetic variability in some automictic populations. The purpose of this paper is to generalize these results to the case where the fitness of an individual is not only a function of its genotype, but also of the density of the population.

\section{ONE Locus Model}

A diploid parthenogenetic population with discrete generations shall be studied which restores diploidy by any combination of the following mechanisms

(1) Inhibition of meiosis I

(2) Inhibition of meiosis II

(3) Central fusion of the haploid egg nuclei

(4) Terminal fusion of the haploid egg nuclei

(5) Gamete duplication (duplication of a single haploid egg nucleus followed by fusion of the cleavage nuclei).

Details of these mechanisms are given in Asher (1970) and Templeton and Rothman (1973). They will not be discussed here except to point out that the genetic consequences of (1) and (3) are identical as are those of (2) and (4).

First, consider a parthenogenetic population with discrete generations consisting of individuals differing at a single locus with two alleles $A$ and $a$. The transition matrix from adults to zygotes in the next generation is given by (Asher, 1972)

\begin{tabular}{|c|c|c|c|c|}
\hline & \multicolumn{3}{|c|}{ Zygotes } \\
\hline & & $A A$ & $a a$ & $A a$ \\
\hline Adults & $\begin{array}{l}A A \\
a a\end{array}$ & $\begin{array}{l}1 \\
0\end{array}$ & $\begin{array}{l}0 \\
1\end{array}$ & $\begin{array}{l}0 \\
0\end{array}$ \\
\hline & $A a$ & $\frac{1}{2}(1-K)$ & $\frac{1}{2}(1-K)$ & $K$ \\
\hline
\end{tabular}

where

$K=E_{1} Y+E_{2}(1-Y / 2)$

$E_{1}=$ the proportion of eggs developing by terminal fusion or inhibition of meiosis II, 
$E_{2}=$ the proportion of eggs developing by central fusion or inhibition of meiosis I,

$E_{3}=$ the proportion of eggs developing by gamete duplication,

$$
E_{1}+E_{2}+E_{3}=1,
$$

$Y=$ the probability of recombination between locus $A$ and its centromere.

Sexual populations reproducing by self-mating are a special case in which $K=\frac{1}{2}$ for all loci, although $K$ can take on values other than one-half if segregation distortion is present (Karlin, 1968).

In order to study the impact of density dependent selection on this system, an absolute fitness will be assigned to each genotype which represents the expected number of offspring of all posible genotypes produced by a zygote with a specific genotype. It is convenient to use a logistic growth model of fitness as Roughgarden (1971) did which defines the fitnesses of the genotypes as

$$
\begin{array}{cc}
\text { Genotypes } & \text { Fitness } \\
A A & r_{A A}+1-\frac{r_{A A}}{C_{A A}} N \\
a a & r_{a a}+1-\frac{r_{a a}}{C_{a a}} N \\
A a & r_{A a}+1-\frac{r_{A a}}{C_{A a}} N
\end{array}
$$

where $N$ is the total population size. The $r+1$ terms represent a density independent or intrinsic component of fitness for each genotype while $(r / C) N$ is a density dependent component of fitness. Throughout this paper both $r$ and $C$ are always assumed positive.

\section{Letting}

$$
\begin{aligned}
& S_{i}=\text { the number of } A A \text { 's at generation } i, \\
& Q_{i}=\text { the number of } a a \text { 's at generation } i, \\
& R_{i}=\text { the number of } A a \text { 's at generation } i, \\
& N_{i}=S_{i}+Q_{i}+R_{i},
\end{aligned}
$$

one obtains the following recursion formulas:

$$
\begin{aligned}
& S_{i+1}=\left[S_{i}+\frac{1}{2}(1-K) R_{i}\right]\left(r_{A A}+1-\left(r_{A A} / C_{A A}\right) N_{i}\right), \\
& Q_{i+1}=\left[Q_{i}+\frac{1}{2}(1-K) R_{i}\right]\left(r_{a a}+1-\left(r_{a a} / C_{a a}\right) N_{i}\right), \\
& R_{i+1}=K R_{i}\left(r_{A a}+1-\left(r_{A a} / C_{A a}\right) N_{i}\right) .
\end{aligned}
$$


Hence,

$$
\begin{aligned}
& \Delta S=\left[S+\frac{1}{2}(1-K) R\right] r_{A A}\left(\left(C_{A A}-S-Q-R\right) / C_{A A}\right)+\frac{1}{2}(1-K) R, \\
& \Delta Q=\left[Q+\frac{1}{2}(1-K) R\right] r_{a a}\left(\left(C_{a a}-S-Q-R\right) / C_{a a}\right)+\frac{1}{2}(1-K) R, \\
& \Delta R=K R r_{A a}\left(\left(C_{A a}-S-Q-R\right) / C_{A a}\right)-(1-K) R .
\end{aligned}
$$

In the special case when only $A A$ individuals are present, (1) reduces to

$$
\Delta S=S r_{A A}\left(C_{A A}-S\right) / C_{A A}
$$

and similarly when only $a a$ individuals are present

$$
\Delta Q=Q r_{a a}\left(C_{a a}-Q\right) / C_{a a} .
$$

The meaning of $r_{A A}, r_{a a}, C_{A A}$ and $C_{a a}$ is now clear. The $r$ 's correspond to the intrinsic finite rates of increase and the $C$ 's to carrying capacities for monomorphic populations of the two types of homozygotes. $C_{A a}$ and $r_{A a}$ receive a similar interpretation in the special case of $K=1$ which occurs when diploidy is restored by central fusion or inhibition of meiosis $I$ and the locus is absolutely linked to the centromere. Otherwise $r_{A a}$ represents the intrinsic finite growth rate of heterozygotes at one generation to all other genotypes produced by them in the next, and $C_{A a}$ represents the population size at which heterozygotes contribute just enough genes to the next generation to replace all the genes they carry.

When the population consists of only the two homozygotes, (1) reduces to

$$
\begin{aligned}
& \Delta S=S r_{A A}\left(C_{A A}-S-Q\right) / C_{A A}, \\
& \Delta Q=Q r_{a a}\left(C_{a a}-S-Q\right) / C_{a a} .
\end{aligned}
$$

This system of equations corresponds to the Gause model of "interspecific" competition with competition coefficients of unity. The system behaves as an interspecific one because the two homozygous genotypes are genetically isolated from one another. Since the competition coefficients are one in this case, the above equations imply that at equilibrium the population will be either entirely $A A$ (which is stable when $C_{A A}>C_{a a}, r_{a a}<2 C_{a a} /\left(C_{A A}-C_{a a}\right)$ and $r_{A A}<2$ ) or $a a$ (when $C_{a a}>C_{A A}, r_{A A}<2 C_{A A} /\left(C_{a a}-C_{A A}\right)$ and $\left.r_{a a}<2\right)$.

When all three genotypes are present the equilibrium solution is obtained by setting the equations in (1) to zero to obtain

$$
\begin{aligned}
N_{e q} & =S_{e q}+Q_{e q}+R_{e q}=C_{A a}\left[1-(1-K) /\left(K r_{A a}\right)\right]=C_{A a e}, \\
S_{e q} & =-\frac{1}{2}(1-K) R_{e q}\left(W_{A A}+1\right) / W_{A A}, \\
Q_{e q} & =-\frac{1}{2}(1-K) R_{e q}\left(W_{a a}+1\right) / W_{a a}, \\
R_{e q} & =C_{A a e}\left[K-\frac{1}{2}(1-K)\left(1 / W_{A A}+1 / W_{a a}\right)\right],
\end{aligned}
$$


where

$$
\begin{aligned}
W_{A A} & =r_{A A}\left(C_{A A}-C_{A a \varepsilon}\right) / C_{A A}, \\
W_{a a} & =r_{a a}\left(C_{a a}-C_{A a \varepsilon}\right) / C_{a a},
\end{aligned}
$$

and $C_{A a e}$ is defined as the effective carrying capacity for a population containing heterozygotes at equilibrium.

The case in which $R_{e q}>0$ is of primary interest for otherwise this case will reduce to (2). First, $P_{e q} \geqslant 0$ and $Q_{e q} \geqslant 0$ implies that $-1 \leqslant W_{A A}<0$ and $-1 \leqslant W_{a a}<0$. This means that the absolute fitnesses, $W_{A A}+1$ and $W_{a a}+1$, of both homozygotes must be less than one at equilibrium so that both homozygotes do not replace themselves. The additional homozygotes needed to yield stationary numbers come from decay into the homozygote classes from the heterozygote class. Also note that $N_{e q}$ is determined solely by the fitness parameters of the heterozygote and the parameter $K$ while the fitnesses of the homozygotes determine how the population is partioned among the genotypes.

The condition $R_{e q}>0$ also requires that $K r_{A a}>1-K$. The quantity $K r_{A a}$ can be thought of as the net intrinsic finite growth rate for heterozygotes from one generation to heterozygotes in the next since $r_{A a}$ is the intrinsic growth rate to all possible genotypes and $K$ is the proportion which arc hetcrozygotes. Similarly, $1-K$ is an intrinsic decay rate from the heterozygote class into the homozygote classes. Hence in order to get $R_{e q}>0$ the net intrinsic growth rate of heterozygotes must exceed the intrinsic decay rate. Another way of stating this inequality is to define the effective intrinsic finite growth rate of heterozygotes as

$$
r_{A a e}=K r_{A a}-(1-K) .
$$

Therefore, to have heterozygotes at equilibrium their effective intrinsic finite rate of natural increase must be greater than zero.

Since it is biologically meaningless to have the expected numbers of offspring $W_{A A}+1$ and $W_{a a}+1$ negative, the $W^{\prime}$ 's may be redefined as

$$
\begin{array}{r}
W_{A A}= \begin{cases}r_{A A}\left(C_{A A}-C_{A a e}\right) / C_{A A} & \text { when }-1<W_{A A}, \\
-1 & \text { when }-1 \geqslant W_{A A},\end{cases} \\
W_{a a}= \begin{cases}r_{a a}\left(C_{a a}-C_{A a e}\right) / C_{a a} & \text { when }-1<W_{a a}, \\
-1 & \text { when }-1 \geqslant W_{a a} .\end{cases}
\end{array}
$$

The conditions that yield $R_{e q} \geqslant 0, S_{e q} \geqslant 0$ and $Q_{e q} \geqslant 0$ are now

$$
\begin{aligned}
r_{A a E} & >0, \\
C_{A u E} & >C_{A A} \text { and } C_{a a} .
\end{aligned}
$$


The effect of $K$, the probability of a heterozygote giving rise to a heterozygote, on (4) is given in Figs. 1 and 2. As can be seen, increasing the value of $K$ broadens the possible range of values of $C_{A A}, C_{a a}$ and $r_{A a}$ that yield $R_{e q}>0$. In this sense factors which increase the value of $K$ enhance the maintenance of heterozygotes.

It is also important to investigate the local stability of the solution given by (3) and (4). It is convenient to make the following transformation:

$$
\begin{aligned}
& z_{1}=S-S_{e q}, \\
& z_{2}=Q-Q_{e q}, \\
& z_{3}=R-R_{e q} .
\end{aligned}
$$

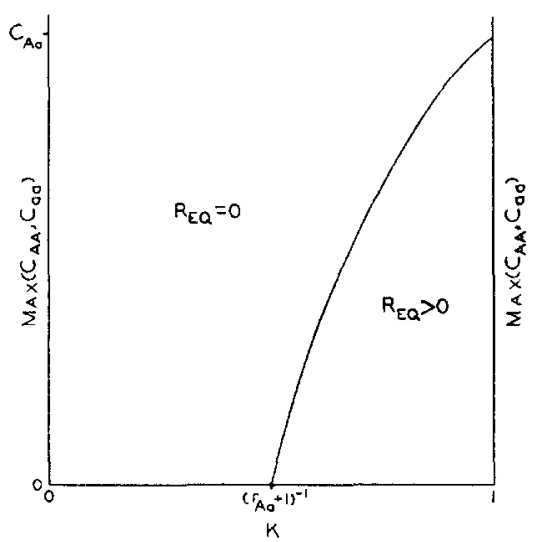

FIG. 1. The effect of $K$, the probability of a heterozygous adult giving rise to a heterozygous zygote, on the range of values of $C_{A A}$ and $C_{a a}$ that yield $R_{e q}>0$ given $C_{A a}$ and $r_{\text {Aae }}>0$.

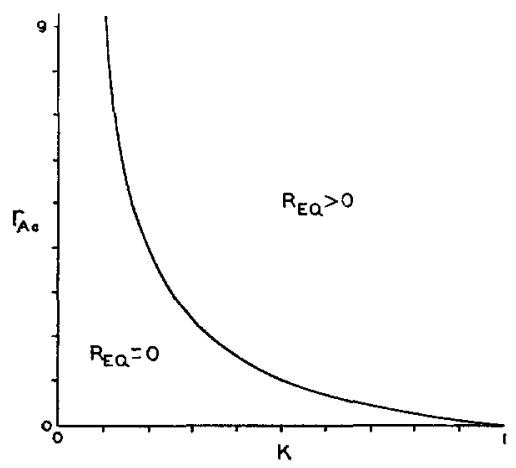

FIG. 2. The effect of $K$ on the ranges of values of $r_{A a}$ that yield $R_{e q}>0$ given $\max \left(C_{A A}, C_{a a}\right)<C_{A a e}$. 
At equilibrium all the $z$ 's are zero. The difference equations for the $z$ 's are calculated by substituting $S=z_{1}+S_{e q}$ and so forth into (1). A linear approximation for these equations in the neighborhood of the equilibrium is obtained by a Taylor's series expansion as

$$
\Delta z=A z
$$

where

$$
\begin{gathered}
z=\left(\begin{array}{l}
z_{1} \\
z_{2} \\
z_{3}
\end{array}\right), \\
A=\left(\begin{array}{ccc}
W_{A A}+Y_{A A} & Y_{A A} & \frac{1}{2}(1-K)\left(W_{A A}+1\right)+Y_{A A} \\
Y_{a a} & W_{a a}+Y_{a a} & \frac{1}{2}(1-K)\left(W_{a a}+1\right)+Y_{u a} \\
\frac{-K r_{A a} R_{e q}}{C_{A a}} & \frac{-K r_{A a} R_{e q}}{C_{A a}} & \frac{-K r_{A a} R_{e q}}{C_{A a}}
\end{array}\right), \\
Y_{A A}=(1-K) R_{e q} r_{A A} /\left(2 W_{A A} C_{A A}\right), \\
Y_{a a}=(1-K) R_{e q} r_{a a} /\left(2 W_{a a} C_{a a}\right) .
\end{gathered}
$$

The solution

$$
z=\left(\begin{array}{l}
0 \\
0 \\
0
\end{array}\right)
$$

will be stable if the eigenvalues of the matrix $A^{*}=A+I$ where $I$ is the identity matrix all lie in the unit circle in the complex plane with center at the origin.

A necessary prerequisite for this stability condition is that the eigenvalues of $A$ must all have negative real parts. The characteristic equation of $A$ is

$$
\lambda^{3}+a_{1} \lambda^{2}+a_{2} \lambda+a_{3}=0
$$

where

$$
\begin{aligned}
a_{1}= & -\left(W_{A A}+W_{a a}+Y_{A A}+Y_{a a}-K r_{A a} R_{e q} / C_{A a}\right), \\
a_{2}= & W_{A A} W_{a a}+R_{e q}\left\{\frac{(1-K)}{2}\left(\frac{r_{a a} W_{A A}}{C_{a a} W_{a a}}+\frac{r_{A A} W_{a a}}{C_{A A} W_{A A}}\right)\right. \\
& \left.-\frac{K r_{A a}}{C_{A a}}\left(\frac{(1-K)}{2}\left(W_{A A}+W_{a a}+2\right)-W_{A A}-W_{a a}\right)\right\}, \\
a_{3}= & W_{A A} W_{a a}\left(K r_{A a}-(1-K)\right) .
\end{aligned}
$$


By the stability criterion of Lienard and Chipart (Gantmacker, 1959) the roots of this equation will have negative real parts when

$$
\begin{aligned}
a_{1} & >0, \\
a_{3} & >0, \\
a_{1} a_{2}-a_{3} & >0 .
\end{aligned}
$$

The conditions that yield $R_{e q}>0$ also yield $a_{1}>0$ and $a_{3}>0$. The last inequality can, after some algebraic manipulations, be rewritten as

$$
r_{A a e}<W_{A A}+W_{a a}+\gamma R_{e q}
$$

where

$$
\begin{aligned}
\gamma= & -\frac{1}{2}(1-K)\left(\frac{r_{A A}}{W_{A A} C_{A A}}+\frac{r_{a a}}{W_{a a} C_{a a}}\right)+K \frac{r_{A a}}{C_{A a}} \\
& +\left\{\frac{1}{2}(1-K)\left(\frac{r_{a a} W_{A A}}{C_{a a} W_{a a}}-\frac{K r_{A a} W_{A A}}{C_{A a}}+\frac{r_{A A} W_{a a}}{C_{A A} W_{a a}}-\frac{K r_{A a} W_{a a}}{C_{A a}}\right)\right. \\
& \left.-\frac{K(1-K) r_{A a}}{C_{A a}}+K \frac{r_{A a}}{C_{A a}}\left(W_{A A}+W_{a a}\right)\right\} \times\left\{1 / W_{A A}+1 / W_{a a}\right. \\
& \left.+R_{e q}\left(\frac{1}{2}(1-K)\left[\frac{r_{A A}}{W_{A A} C_{A A}}+\frac{r_{a a}}{W_{a a} C_{a a}}\right]-K \frac{r_{A a}}{C_{A a}}\right)\right\} .
\end{aligned}
$$

The conditions that yield $R_{e q}>0$ insure that $\gamma>0$ and in general $\gamma R_{e q}$ will be a large, positive number. Since $W_{A A}+W_{a a}$ can only vary from 0 to -2 , the number $W_{A A}+W_{a a}+\gamma R_{e q}$ will also in general be a large, positive number. In realistic situations $r_{A a e}$ will be small, (probably little more than one or two at the most) so that inequality (5) will add no additional restrictions in most situations.

It now remains to be shown that $|\lambda+1|<1$ for all the eigenvalues, $\lambda$, of $A$. Unfortunately, all attempts to prove in general that the eigenvalues of $A$ satisfy this inequality have proven unsuccessful. However, some insight into the additional stability restrictions needed can be obtained from studying the special case in which both homozygotes liave the same fitnesses. Substituting $r_{A A}=r_{a a}=r_{h}, C_{A A}=C_{a a}=C_{h}$ and $\lambda^{*}=\lambda-1$ into the characteristic equation of $A$ yields the characteristic equation of $A^{*}$ for this special case to be

$$
\begin{gathered}
\left(W_{h}+1-\lambda^{*}\right)\left(W_{h}+2 Y_{h}+1-\lambda^{*}\right)\left(1-K r_{A a} R_{e q} / C_{A a}-\lambda^{*}\right) \\
-2 K r_{A a} R_{e q}\left[\frac{1}{2}(1-K)\left(W_{h}+1\right)+Y_{h}\right] / C_{A a}
\end{gathered}
$$


where

$$
\begin{aligned}
W_{h} & =r_{h}\left(C_{h}-C_{A a e}\right) / C_{h}, \\
Y_{h} & =\frac{1}{2}(1-K) R_{e q} r_{h} /\left(W_{h} C_{h}\right) .
\end{aligned}
$$

One eigenvalue of $A^{*}$ for this special case is therefore $\lambda_{1}{ }^{*}=W_{h}+1$ which is always less than one in absolute value under conditions that yield $R_{e q}>0$. The other two eigenvalues are the roots of

$$
\lambda^{*^{2}}+a_{1} \lambda^{*}+a_{2}=0
$$

where

$$
\begin{aligned}
& a_{1}=\left(K R_{e q} r_{A a} / C_{A a}\right)-2-W_{h}-2 Y_{h} \\
& a_{2}=1+W_{h}+2 Y_{h}-\left(K^{2} R_{e q} r_{A a}\left(W_{h}+1\right) / C_{A a}\right) .
\end{aligned}
$$

The inequality $\left|\lambda^{*}\right|<1$ will be satisfied for both roots when (Goldberg, 1958)

$$
\begin{array}{r}
1+a_{1}+a_{2}>0 \\
1-a_{1}+a_{2}>0 \\
1-a_{2}>0 .
\end{array}
$$

These inequalities will be satisfied only when the previously discussed stability conditions hold and in addition when

$$
\begin{aligned}
r_{h} & <\left(\frac{1-K}{2+W_{h}}\right)\left(\frac{R_{e q}}{C_{A a e}-C_{h}}\right), \\
r_{A a e} & <\left(\frac{C_{A a}}{R_{e q}}\right)\left(\frac{4+2\left(W_{h}+2 Y_{h}\right)}{1+K\left(W_{h}+1\right)}\right)-(1-K) .
\end{aligned}
$$

Therefore, additional restrictions upon the magnitudes of the finite rates of increase must be made. These additional restrictions are needed because if the $r$ 's are too large, population growth need not be smooth, and oscillations in population size could occur that lead to local instability. These oscillations arise in a discrete model because the population may overshoot its carrying capacity in the intervals between application of the density-dependent control.

\section{Two Locus Model}

The results of the preceding section can be extended to two loci with two alleles at each locus. The general form of the transition matrix from adults to zygotes for a parthenogenetic population reproducing by any combination of the 
mechanisms previously discussed and for a self-mating sexual population is (Asher, 1972)

Zygotes

$A B / A B \quad A b / A b \quad a B / a B \quad a b / a b \quad A B / a B \quad A b / a b \quad A B / A b \quad a B / a b \quad A B / a b \quad A b / a b$

\begin{tabular}{|c|c|c|c|c|c|c|c|c|c|}
\hline$A B / A B$ & 1 & 0 & 0 & 0 & 0 & 0 & 0 & 0 & 0 \\
\hline$A b / A b$ & 0 & 1 & 0 & 0 & 0 & 0 & 0 & 0 & 0 \\
\hline$a B / a B$ & 0 & 0 & 1 & 0 & 0 & 0 & 0 & $\mathbf{0}$ & 0 \\
\hline$a b / a b$ & 0 & 0 & 0 & 1 & 0 & 0 & 0 & 0 & 0 \\
\hline$A B / a B$ & $\frac{1-K^{\prime}}{2}$ & 0 & $\frac{1-K^{\prime}}{2}$ & 0 & $K^{\prime}$ & 0 & 0 & 0 & 0 \\
\hline$A b / a b$ & 0 & $\frac{1-K^{\prime}}{2}$ & 0 & $\frac{1-K^{\prime}}{2}$ & 0 & $K^{\prime}$ & 0 & 0 & 0 \\
\hline$A B / A b$ & $\frac{1-K^{\prime \prime}}{2}$ & $\frac{1-K^{\prime \prime}}{2}$ & 0 & 0 & 0 & 0 & $K^{\prime \prime}$ & 0 & 0 \\
\hline$a B / a b$ & 0 & $\mathbf{0}$ & $\frac{1-K^{\prime \prime}}{2}$ & $\frac{1-K^{\prime \prime}}{2}$ & 0 & 0 & 0 & $K^{\prime \prime}$ & 0 \\
\hline$A B / a b$ & $\alpha_{1}$ & $\alpha_{2}$ & $\alpha_{2}$ & $\alpha_{1}$ & $\alpha_{3}$ & $\alpha_{3}$ & $\alpha_{4}$ & $\alpha_{4}$ & $\alpha_{5}$ \\
\hline$A b / a B$ & $\alpha_{2}$ & $\alpha_{1}$ & $\alpha_{1}$ & $\alpha_{2}$ & $\alpha_{3}$ & $\alpha_{3}$ & $\alpha_{4}$ & $\alpha_{4}$ & $u_{6}$ \\
\hline
\end{tabular}

The actual values of these parameters are given in Table $\mathrm{I}$ in terms of $E_{1}, E_{2}$, $E_{3}$ and the map distances between the loci and between a locus and itscentromere. The coefficients of coincidence are assumed to be one, so the recombination frequency is given by

$$
r=\frac{1}{2}\left(1-e^{-2 x}\right)
$$

where

$$
\begin{aligned}
& r=\text { the recombination frequency, } \\
& x=\text { the map distance }
\end{aligned}
$$

and the probability of recombination $Y$ is given by (Barratt et al., 1954)

$$
Y=\frac{2}{3}\left(1-e^{-3 x}\right) .
$$


DENSITY DEPENDENT \$ELECTION

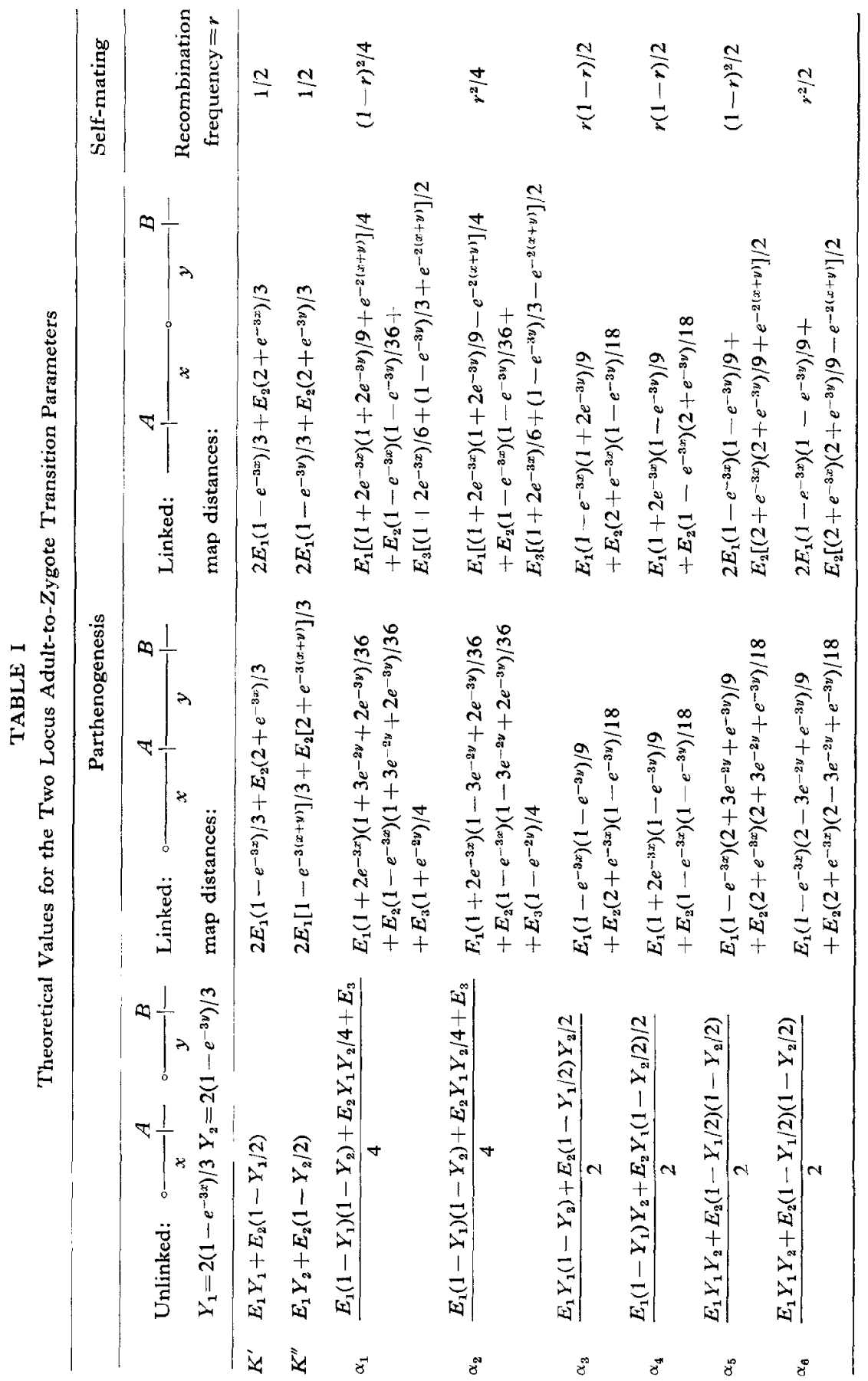


Define

$$
\begin{aligned}
& X_{1}=\text { the number of } A B / A B \text { individuals; } \\
& X_{2}=\text { the number of } A b / A b \text { individuals; } \\
& X_{3}=\text { the number of } a B / a B \text { individuals; } \\
& X_{4}=\text { the number of } a b / a b \text { individuals; } \\
& X_{5}=\text { the number of } A B / a B \text { individuals; } \\
& X_{6}=\text { the number of } A b / a b \text { individuals; } \\
& X_{7}=\text { the number of } A B / A b \text { individuals; } \\
& X_{8}=\text { the number of } a B / a b \text { individuals; } \\
& X_{9}=\text { the number of } A B / a b \text { individuals; } \\
& X_{10}=\text { the number of } A b / a B \text { individuals. }
\end{aligned}
$$

The fitnesses for the various genotypes will be

$$
\begin{gathered}
r_{i}+1-\left(r_{i} / C_{i}\right) N \quad i=1,2, \ldots, 10 \\
N=\sum_{i=1}^{10} X_{i} .
\end{gathered}
$$

The fitnesses of the cis and trans double heterozygotes are assumed to be equal, so $r_{9}=r_{10}$ and $C_{9}=C_{10}$.

The set of fundamental difference cquations is

$$
\begin{aligned}
\Delta X_{1}= & \left(X_{1}+\frac{1-K^{\prime}}{2} X_{5}+\frac{1-K^{\prime \prime}}{2} X_{7}+\alpha_{1} X_{9}+\alpha_{2} X_{10}\right) r_{1} \frac{C_{1}-N}{C_{1}} \\
& +\frac{1-K^{\prime}}{2} X_{5}+\frac{1-K^{\prime \prime}}{2} X_{7}+\alpha_{1} X_{9}+\alpha_{2} X_{10}, \\
\Delta X_{2}= & \left(X_{2}+\frac{1-K^{\prime}}{2} X_{6}+\frac{1-K^{\prime \prime}}{2} X_{7}+\alpha_{2} X_{9}+\alpha_{1} X_{10}\right) r_{2} \frac{C_{2}-N}{C_{2}} \\
& +\frac{1-K^{\prime}}{2} X_{6}+\frac{1-K^{\prime \prime}}{2} X_{7}+\alpha_{2} X_{9}+\alpha_{1} X_{10}, \\
\Delta X_{3}= & \left(X_{3}+\frac{1-K^{\prime}}{2} X_{5}+\frac{1-K^{\prime \prime}}{2} X_{8}+\alpha_{2} X_{9}+\alpha_{1} X_{10}\right) r_{3} \frac{C_{3}-N}{C_{3}} \\
& +\frac{1-K^{\prime}}{2} X_{5}+\frac{1-K^{\prime \prime}}{2} X_{8}+\alpha_{2} X_{9}+\alpha_{1} X_{10}, \\
\Delta X_{4}= & \left(X_{4}+\frac{1-K^{\prime}}{2} X_{6}+\frac{1-K^{\prime \prime}}{2} X_{8}+\alpha_{1} X_{9}+\alpha_{2} X_{10}\right) r_{4} \frac{C_{4}-N}{C_{4}} \\
& +\frac{1-K^{\prime}}{2} X_{6}+\frac{1-K^{\prime \prime}}{2} X_{8}+\alpha_{1} X_{9}+\alpha_{2} X_{10},
\end{aligned}
$$




$$
\begin{aligned}
& \Delta X_{5}=\left(K^{\prime} X_{5}+\alpha_{3} X_{9}+\alpha_{3} X_{10}\right) r_{5} \frac{C_{5}-N}{C_{5}}-\left(1-K^{\prime}\right) X_{5}+\alpha_{3}\left(X_{9}+X_{10}\right), \\
& \Delta X_{6}=\left(K^{\prime} X_{6}+\alpha_{3} X_{9}+\alpha_{3} X_{10}\right) r_{6} \frac{C_{6}-N}{C_{6}}-\left(1-K^{\prime}\right) X_{6}+\alpha_{3}\left(X_{9}+X_{10}\right), \\
& \Delta X_{7}=\left(K^{\prime \prime} X_{7}+\alpha_{4} X_{9}+\alpha_{4} X_{10}\right) r_{7} \frac{C_{7}-N}{C_{7}}-\left(1-K^{\prime \prime}\right) X_{7}+\alpha_{4}\left(X_{9}+X_{10}\right), \\
& \Delta X_{8}=\left(K^{\prime \prime} X_{8}+\alpha_{4} X_{9}+\alpha_{4} X_{10}\right) r_{8} \frac{C_{8}-N}{C_{8}}-\left(1-K^{\prime \prime}\right) X_{8}+\alpha_{4}\left(X_{9}+X_{10}\right), \\
& \Delta X_{9}=\left(\alpha_{5} X_{9}+\alpha_{6} X_{10}\right) r_{9} \frac{C_{9}-N}{C_{9}}-\left(1-\alpha_{5}\right) X_{9}+\alpha_{6} X_{10}, \\
& \Delta X_{10}=\left(\alpha_{6} X_{9}+\alpha_{5} X_{10}\right) r_{9} \frac{C_{9}-N}{C_{9}}+\alpha_{6} X_{9}-\left(1-\alpha_{5} X_{10}\right) .
\end{aligned}
$$

The outcomes of competition among various genotypic arrays in which double heterozygotes are not present is given in Table II. When the double heterozygotes are present in the population, the equilibrium solution obtained by setting (6) equal to zero is:

$$
\begin{aligned}
& N_{e q}=C_{9}\left[1-\frac{1-\alpha_{5}-\alpha_{6}}{\left(\alpha_{5}+\alpha_{6}\right) r_{9}}\right]=C_{9 e}, \\
& X_{9}=X_{10} \\
& X_{8}=-\frac{2 \alpha_{4} X_{9}\left(W_{8}+1\right)}{K^{\prime \prime} W_{8}-\left(1-K^{\prime \prime}\right)}, \\
& X_{7}=-\frac{2 \alpha_{4} X_{9}\left(W_{7}+1\right)}{K^{\prime \prime} W_{7}-\left(1-K^{\prime \prime}\right)}, \\
& X_{6}=-\frac{2 \alpha_{3} X_{9}\left(W_{6}+1\right)}{K^{\prime} W_{6}-\left(1-K^{\prime \prime}\right.}, \\
& X_{5}=-\frac{2 \alpha_{3} X_{9}\left(W_{5}+1\right)}{K^{\prime} W_{5}-\left(1-K^{\prime}\right)}, \\
& X_{4}=\left[\frac{\left(1-K^{\prime}\right) \alpha_{3}\left(W_{6}+1\right)}{K^{\prime} W_{6}-\left(1-K^{\prime}\right)}+\frac{\left(1-K^{\prime \prime}\right) \alpha_{4}\left(W_{8}+1\right)}{K^{\prime \prime} W_{8}-\left(1-K^{\prime \prime}\right)}-\left(\alpha_{1}+\alpha_{2}\right)\right] \frac{\left(W_{4}+1\right) X_{9}}{W_{4}}, \\
& X_{3}=\left[\frac{\left(1-K^{\prime}\right) \alpha_{3}\left(W_{5}+1\right)}{K^{\prime} W_{5}-\left(1-K^{\prime}\right)}+\frac{\left(1-K^{\prime \prime}\right) \alpha_{4}\left(W_{8}+1\right)}{K^{\prime \prime} W_{8}-\left(1-K^{\prime \prime}\right)}-\left(\alpha_{1}+\alpha_{2}\right)\right] \frac{\left(W_{3}+1\right) X_{9}}{W_{3}}, \\
& X_{2}=\left[\frac{\left(1-K^{\prime}\right) \alpha_{3}\left(W_{6}+1\right)}{K^{\prime} W_{6}-\left(1-K^{\prime}\right)}+\frac{\left(1-K^{\prime \prime}\right) \alpha_{4}\left(W_{7}+1\right)}{K^{\prime \prime} W_{7}-\left(1-K^{\prime \prime}\right)}-\left(\alpha_{1}+\alpha_{2}\right)\right] \frac{\left(W_{2}+1\right) X_{9}}{W_{2}},
\end{aligned}
$$




$$
\begin{aligned}
& X_{1}=\left[\frac{\left(1-K^{\prime}\right) \alpha_{3}\left(W_{5}+1\right)}{K^{\prime} W_{5}-\left(1-K^{\prime}\right)}+\frac{\left(1-K^{\prime \prime}\right) \alpha_{4}\left(W_{7}+1\right)}{K^{\prime \prime} W_{7}-\left(1-K^{\prime \prime}\right)}-\left(\alpha_{1}+\alpha_{2}\right)\right] \frac{\left(W_{1}+1\right) X_{9}}{W_{1}} \\
& X_{9}=\frac{C_{9 e}}{\left(\begin{array}{c}
2-2 \alpha_{4} b_{1}-2 \alpha_{3} b_{2} \\
\left.-\left(\alpha_{1}+\alpha_{2}\right)\left[\frac{W_{4}+1}{W_{4}}+\frac{W_{3}+1}{W_{3}}+\frac{W_{2}+1}{W_{2}}+\frac{W_{1}+1}{W_{1}}\right]\right)
\end{array}\right.}
\end{aligned}
$$

TABLE II

Outcomes of Competition with the Two Locus Model When

\begin{tabular}{|c|c|c|}
\hline $\begin{array}{l}\text { Competing } \\
\text { genotypes }\end{array}$ & $\begin{array}{l}\text { Equilibrium } \\
\text { population }\end{array}$ & Stability Conditions \\
\hline $\begin{array}{l}X_{i} i=1,2,3,4 \\
\text { (two locus } \\
\text { homozygotes) }\end{array}$ & $\begin{array}{l}N_{e q}=X_{1 ? q}=C_{1} \\
X_{i}=0 \quad i=2,3,4\end{array}$ & $\begin{array}{l}C_{1}>C_{2}, C_{\mathrm{a}}, C_{4} \\
r_{1}<2 \\
r_{i}<2 C_{i} /\left(C_{1}-C_{i}\right) \quad i=2,3,4\end{array}$ \\
\hline $\begin{array}{l}X_{i} i=1, \ldots, 8 \\
\text { (two locus } \\
\text { homozygotes } \\
\text { and single } \\
\text { locus } \\
\text { heterozygotes) }\end{array}$ & $\begin{array}{l}N_{e q}=X_{1 e q}=C_{1} \\
X_{i}=0 \quad i=2, \ldots, 8\end{array}$ & $\begin{array}{l}C_{1}>C_{2}, C_{3}, C_{4} \\
C_{1}>C_{j e}=C_{j}\left[1-\left(1-K^{\prime}\right) /\left(r_{j} K^{\prime}\right)\right] j=5,6 \\
C_{1}>C_{u e}=C_{u}\left[1-\left(1-K^{\prime \prime}\right) /\left(r_{u} K^{\prime \prime}\right)\right] u=7,8 \\
r_{1}<2 \\
r_{i}<2 C_{i} /\left(C_{1}-C_{i}\right) i=2,3,4 \\
r_{j}<\left(1+K^{\prime}\right) C_{j} /\left[K^{\prime}\left(C_{1}-C_{j}\right)\right] j=5,6 \\
r_{u}<\left(1+K^{\prime \prime}\right) C_{u} /\left[K^{\prime \prime}\left(C_{1}-C_{i k}\right)\right] u=7,8\end{array}$ \\
\hline $\begin{array}{l}X_{i} i=1, \ldots, 8 \\
\text { (two locus } \\
\text { lonnozygoles } \\
\text { and single } \\
\text { locus } \\
\text { heterozygotes) }\end{array}$ & $\begin{array}{l}N_{e q}=C_{5 e} \\
X_{5 e q}=C_{5 e} / M^{a} \\
X_{1 e q}=G_{i}^{b} i-1,3 \\
X_{i}=0 i=2,4,6,7,8\end{array}$ & $\begin{array}{l}C_{5 e}>C_{1}, C_{2}, C_{3}, C_{4}, C_{6 e}, C_{7 e}, C_{8 e} \\
r_{i}<2 C_{i} /\left(C_{5 e}-C_{i}\right) i=2,4 \\
r_{h}<\left(1-K^{\prime}\right) X_{5 e q} /\left[\left(2+W_{h}\right)\left(C_{5 e}-C_{h}\right)\right]^{c} \\
r_{6}<(1+K) C_{6} /\left[K^{\prime}\left(C_{5 e}-C_{6}\right)\right] \\
r_{u}<\left(1+K^{\prime \prime}\right) C_{u} /\left[K^{\prime \prime}\left(C_{5 e}-C_{u}\right)\right] u=7,8 \\
0<r_{5 e}=K^{\prime} r_{5}-\left(1-K^{\prime}\right)<W_{1}+W_{3}+\gamma_{5} X_{5 e .}{ }^{a} \\
r_{5 e}<\frac{C_{5 e}}{X_{5 e q}} \frac{4+2\left(W_{h}+Y_{h}\right)}{1+K^{\prime}\left(W_{1}+1\right)}-\left(1-K^{\prime}\right)^{c}\end{array}$ \\
\hline
\end{tabular}

Double Heterozygotes Are Not Present

a $M=K^{\prime}-\frac{1}{2}\left(1-K^{\prime}\right)\left(1 / W_{1}+1 / W_{3}\right)$ where $W_{1}=r_{1}\left(C_{1}-C_{5 e}\right) / C_{1}$ and $W_{3}=r_{3}\left(C_{3}-C_{5 e}\right) / C_{3}$.

${ }^{b} G_{i}--\frac{1}{2}\left(1-K^{\prime}\right) X_{\text {seq }}\left(W_{i}+1\right) / W_{i}$.

${ }^{c}$ Stability has only been shown when $r_{1}=r_{3}=r_{h}, C_{1}=C_{3}=C_{h}, W_{h}=r_{h}\left(C_{h}-C_{5 e}\right) / C_{h}$ and $Y_{h}=\left(1-K^{\prime}\right) X_{5 e q} /\left(C_{h} W_{h}\right)$.

a $\gamma_{5}$ is defined in a manner analogous to the one locus $\gamma$. 
where

$$
\begin{aligned}
W_{i}=r_{i}\left(C_{i}-C_{9 e}\right) / C_{i}, \\
b_{1}=\frac{W_{8}+1}{K^{\prime \prime} W_{8}-\left(1-K^{\prime \prime}\right)}\left[1-\left(1-K^{\prime \prime}\right)\left(\frac{W_{4}+1}{W_{4}}+\frac{W_{3}+1}{W_{3}}\right)\right] \\
+\frac{W_{7}+1}{K^{\prime \prime} W_{7}-\left(1-K^{\prime \prime}\right)}\left[1-\left(1-K^{\prime \prime}\right)\left(\frac{W_{2}+1}{W_{2}}+\frac{W_{1}+1}{W_{1}}\right)\right], \\
b_{2}=\frac{W_{6}+1}{K^{\prime} W_{6}-\left(1-K^{\prime}\right)}\left[1-\left(1-K^{\prime}\right)\left(\frac{W_{4}+1}{W_{4}}+\frac{W_{2}+1}{W_{2}}\right)\right] \\
+\frac{W_{5}+1}{K^{\prime} W_{5}-\left(1-K^{\prime}\right)}\left[1-\left(1-K^{\prime}\right)\left(\frac{W_{3}+1}{W_{3}}+\frac{W_{1}+1}{W_{1}}\right)\right] .
\end{aligned}
$$

Once again the total population size is determined only by the fitness of the double heterozygotes, while the partitioning of the population among the various genotypes is determined by the fitnesses at equilibrium of all the other genotypes.

The case in which $X_{9}$ and $X_{10}$ are greater than zero is of primary interest for otherwise this will reduce to one of the cases given in Table II. The conditions that yield $X_{9}, X_{10}>0$ at equilibrium are solved from (7) as

$$
\begin{gathered}
r_{9}\left(\alpha_{5}+\alpha_{6}\right)-\left(1-\alpha_{5}-\alpha_{6}\right)=r_{9 e}>0, \\
C_{9 e}>C_{i}, C_{j e}, \quad i=1,2,3,4, \quad j=5,6,7,8 .
\end{gathered}
$$

These conditions are analogous to those given in the one locus model since $\alpha_{5}+\alpha_{6}$ is the probability of a double heterozygote giving rise to a double heterozygote; therefore, $r_{9}\left(\alpha_{5}+\alpha_{6}\right)$ represents the net finite intrinsic growth rate for double heterozygotes, $1-\alpha_{5}-\alpha_{6}$ the intrinsic decay rate and $r_{9 e}$ the effective finite intrinsic growth rate of double heterozygotes. Similarly, $C_{\mathbf{g}_{e}}$ is of a form completely analogous to $C_{A a e}$ in the one locus model. Thus the biological meaning of these conditions is identical to the meaning of (4).

Unfortunately, it has not been possible to determine the stability of this solution. However, the close similarity of the mathematical and biological meanings of the conditions that result in a nontrivial equilibrium in this case to those of the one locus case strongly suggest that this is also a stable equilibrium.

\section{Extension to $n$ Loci}

Let $g n$ represent a genotype that is heterozygous at $n$ loci. A proportion, $1-P_{n}$, of the zygotes produced by an adult with genotype $g n$ will lose heterozygosity at one or more loci while $P_{n}$ of them will retain heterozygosity at all $n$ 
loci. Finally, let $g n$ be in a population consisting of several genotypes, but in which $g n$ is the genotype with maximum heterozygosity. Zygotes with less than $n$ heterozygous loci could be produced either by adults with identical genotypes in the previous generation or from decay from the more heterozygous genotypes. However, no other genotype can decay into $g n$, so all $g n$ individuals must have been produced by a $g n$ adult in the previous generation. Hence, if the fitness of $g n$ is $r_{n}+1-r_{n} N / C_{n}$ where $N$ is the total population size, then

$$
\Delta X_{n}=P_{n} X_{n} r_{n}\left(\left(C_{n}-N\right) / C_{n}\right)-\left(1-P_{n}\right) X_{n}
$$

where $X_{n}$ is the number of $g n$ individuals. Thus at equilibrium

$$
N_{e q}=C_{n}\left(1-\left(1-P_{n}\right) /\left(r_{n} P_{n}\right)\right)=C_{n e} .
$$

The above quantity will be greater than zero only if

$$
r_{n} P_{n}-\left(1-P_{n}\right)=r_{n e}>0 .
$$

I must now consider how gn interacts with the other genotypes in such a manner to yield $X_{n}>0$ at equilibrium. For those genotypes that are genetically isolated from $g n$, i.e., those that $g n$ cannot decay into, the competitive exclusion principle tells us that such genotypes will become extinct if the equilibrium population with them present and $g n$ absent is less than $C_{n e}$. For those genotypes into which $g n$ can decay the following situation holds.

First, since some of the zygotes produced by $g n$ individuals will be of these alternative genotypes, it is obvious that an equilibrium with $X_{n}>0$ cannot occur unless the fitnesses of the other genotypes are such that at equilibrium these genotypes do not completely replace themselves. The additional members of these other genotypes needed to yield stationary numbers come from decay from the more heterozygous genotypes. This can be expressed mathematically by letting $P_{i}$ be the proportion of zygotes with genotype $i$ produced by an adult with genotypc $i$. Consequently $1-P_{i}$ of the zygotes produced by genotype $i$ are of a different genotype and represent an intrinsic decay rate for $i$. The stipulation that at equilibrium the fitnesses must be such that the $i$ th genotype does not replace itself is

$$
\begin{gathered}
P_{i} r_{i}\left(C_{i}-C_{n e}\right) / C_{i}<1-P_{i}, \\
C_{n e}>C_{i}\left(1-\left(1-P_{i}\right) /\left(P_{i} r_{i}\right)\right)=C_{i e} .
\end{gathered}
$$

This definition of $C_{i e}$ is applicable even when $i$ represents a totally homozygous genotype for in that case $P_{i}=1$, so $C_{i e}-C_{i}$ and $r_{i e}=r_{i}$. 


\section{Extension to Nonlogistic Fitness Functions}

In this section the absolute fitness of genotype $i$ is assumed to be

$$
1+W_{i}(N)
$$

where $W_{i}(N)$ is a function of the genotype $i$ and of the population size $N$. In order to impose a limit on population growth, it is further assumed that $W_{i}(N)$ is a strictly decreasing function of $N$, as was done by Charlesworth (1971) for a one-locus model of a random-mating population.

As before, let $g n$ be a genotype heterozygous at $n$ loci, and let $g n$ be present in a population in which it represents the genotype with maximum heterozygosity. Then, using (8),

$$
\Delta X_{n}=P_{n} X_{n} W_{n}(N)-\left(1-P_{n}\right) X_{n}
$$

At equilibrium,

$$
N_{e a}=f_{n}\left[\left(1-P_{n}\right) / P_{n}\right]=C_{n e}^{\prime}
$$

where $f_{n}$ represents the inverse function of $W_{n}$ such that $f_{n}\left[W_{n}(N)\right]=N$. Because $f_{n}\left[\left(1-P_{n}\right) / P_{n}\right]$ represents the final size of a population in which $g n$ is present at equilibrium, it can also be thought of as an effective carrying capacity. Note that, just as in the logistic case, this effective carrying capacity is determined solely by the fitness function for genotype $g n$ and by $P_{n}$.

Necessary conditions for $X_{n}>0$ at equilibrium are

$$
\begin{aligned}
& C_{n e}^{\prime}=f_{n}\left\{\left(1-P_{n}\right) / P_{n}\right\}>0, \\
& C_{n e}^{\prime}>f_{j}\left\{\left(1-P_{j}\right) / P_{j}\right\}=C_{j e}^{\prime} \quad \text { for all other genotypes } j .
\end{aligned}
$$

The first inequality reduces to $r_{n e}>0$ in the logistic case, while the second inequality insures that the fitnesses of all other genotypes $j$ are such that they do not completely replace themselves at equilibrium. This last inequality shows that, just as in the logistic casc, the equilibrium population consists of that ensemble of genotypes which maximizes population size. Thus, the qualitative aspects of the logistic fitness model are true whenever fitness is a decreasing function of density.

\section{Discussion}

As can be seen for the situations discussed in this paper, the following is true:

$$
\begin{aligned}
& N_{e q}=C_{n e}, \\
& C_{n e}>C_{i e} \quad \text { for all other genotypes } i, \\
& r_{n e}>0,
\end{aligned}
$$


where

$$
\begin{aligned}
C_{i e}= & C_{i}\left(1-\frac{1-P_{i}}{P_{i} r_{i}}\right) \quad \text { for all genotypes, } \\
r_{i e}= & r_{i} P_{i}-\left(1-P_{i}\right) \quad \text { for all genotypes, } \\
P_{i}= & \text { the proportion of zygotes of genotype } \\
& i \text { among all zygotes produced by an } \\
& \text { adult of genotype } i,
\end{aligned}
$$

and $n$ is the largest number of loci for which a genotype is heterozygous in the equilibrium population. This equilibrium solution is very similar to the equilibrium solution in a model of interspecific competition within a common niche. If a group of species was competing within a common niche, at equilibrium only one species, say $y$, would survive such that

$$
\begin{aligned}
N_{e a} & =C_{y}, \\
C_{y} & >C_{i} \quad \text { for all other species } i, \\
r_{y} & >0,
\end{aligned}
$$

where $C$ and $r$ refer to the carrying capacities and intrinsic growth rates of genetically isolated and homogeneous species. As can be seen, (11) is identical in form to (10). Thus, the complexity of the genetically heterogeneous population which involves segregation, recombination and mode of reproduction can be summarized in the parameter $P_{i}$. Then $C_{i e}$ and $r_{i e}$ which are functions of $P_{i}$ and the logistic fitness parameter reduce the problem of compctition in a genetically heterogeneous population to an equivalent case of competition between genetically isolated, homogeneous populations with respect to the equilibrium solution. In this sense, $C_{i e}$ and $r_{i e}$ play a role similar to that of $N_{e}$, the effective population size, in population genetics. All reduce complicated situations to an equivalent ideal case with respect to certain parameters of interest.

However, it should be emphasized that this analogy is valid only with respect to the equilibrium solution since the "type" of equilibrium population which corresponds to the "species" in the analogy becomes meaningful only at equilibrium. For example, it is erroneous to say that a population of $A A, a a$ and $A a$ individuals is competing against a population of $A A$ individuals. Obviously the true competition is between the individuals of the various genotypes and not between types of equilibrium populations. Also, once at equilibrium there will in general still be competition between genotypes since the equilihrium population can be genetically heterogeneous.

In all cases where heterozygotes were present at equilibrium the fitnesses were such that there was heterosis as manifested through the effective carrying 
capacities. In the absence of heterosis, heterozygotes will become extinct. This extends the conclusions of Asher (1972) based on constant fitness models and selection in changing environments that "... heterozygostiy in automictic parthenogenetic and self-mating populations may only exist as a consequence of selection." Thus, the very presence of heterozygotes in such populations can be regarded as evidence for heterosis.

In this model, "heterosis" is manifested through the $C_{n e}^{\prime}$ 's and occurs when populations with heterozygotes are larger than those without heterozygotes since selection operates to maximize population size by choosing that ensemble of genotypes with the largest $C_{n e}^{\prime}$. Heterosis in this model also means that the genotype with maximum heterozygosity has the highest relative fitness at equilibrium since the fitness of any given genotype, say $i$, relative to the maximum heterozygote is

$$
\left(1+W_{i}\left(N_{e q}\right)\right) /\left(1+W_{n}\left(N_{e q}\right)\right)
$$

which is less than one for $i \neq n$ whenever $C_{n e}^{\prime}>C_{i c}^{\prime}$.

In all cases this largest $C_{n e}^{\prime}$ can be written as $f_{n}\left\{\left(1-P_{n}\right) / P_{n}\right\}$ and in general $P_{n}<1$ for heterozygous genotypes. As can be seen from (9), increasing the valuc of $P_{n}$ will increase the value of $N_{e q}$ since $W_{n}$ is a decreasing function of $N$. Therefore, population size can be further increased if $P$ can be increased through the action of selection. Such increases in $P$ would be favored under the model considered here if heterosis were present since the highest ranking heterozygote has the largest relative fitness. Furthermore, as $P_{n}$ goes to one, $W_{n}\left(N_{e q}\right)$ must go to zero so that the relative fitnesses of all the other genotypes go to $1+W_{i}\left(N_{e q}\right)$. As $P_{n}$ gets larger, $N_{e q}$ becomes larger and $W_{i}\left(N_{e q}\right)$ decreases. Consequently, the fitnesses of all other genotypes relative to the fitness of the maximal heterozygote decreases as $P_{n}$ goes to one. As these relative fitnesses decrease, the selective forces driving $P_{n}$ towards one increase. Thus, such populations represent a system which, if primed with an initial amount of heterosis, tends to become locked in on a heterotic strategy and evolves more and more heterosis. ${ }^{1}$ A consequence of such " $P$ " selection is that certain types of automictic parthenogenetic populations can exploit the microevolutionary advantages of heterosis more efficiently than sexual populations, but with perhaps the long term disadvantage that they tend to become locked into a single, adaptive strategy.

As can be seen in the one and two locus cases were the $P$ 's are explicitly stated, the $P$ 's are functions of the frequencies of the diploid restoring mechanisms and the linkage relations. These relations are shown graphically for the one locus model in Fig. 3. Both the method of restoring diploidy and linkage relations can be theoretically modified. For example, parthenogenetic strains

${ }^{1}$ A more rigorous proof of this and similar effects will be the subject of a subsequent paper. 
of Drosophila mercatorum restore diploidy by gamete duplication and central and/or terminal fusion (Carson et al., 1969). Since gamete duplication produces only homozygotes, the greater $E_{3}$, the proportion of gamete duplication, the smaller will be the $P$ 's for heterozygous genotypes. Thus, given some initial heterosis, any genetic change which decreases $E_{3}$ will be favored. It is therefore important to see if $E_{3}$ can be modified genetically. Carson (personal communication) has recently shown that the value of $E_{3}$ varies from $99 \%$ to $78 \%$ in various strains of $D$. mercatorum. This suggests that $E_{3}$ is under genetic control to some extent and that genetic variability with respect to this trait exists. Consequently it would be possible to modify $E_{3}$ in this species through selection favoring heterozygous genotypes.

A possible example of this type of $\mathbf{P}$-selection may be found in the naturally occuring parthenogenetic fly Drosophila mangabeirai. Drosophila mangabeirai reproduces primarily by central fusion (Murdy and Carson, 1959) which in general results in the highest $P$ values for heterozygous genotypes (see Fig. 3).

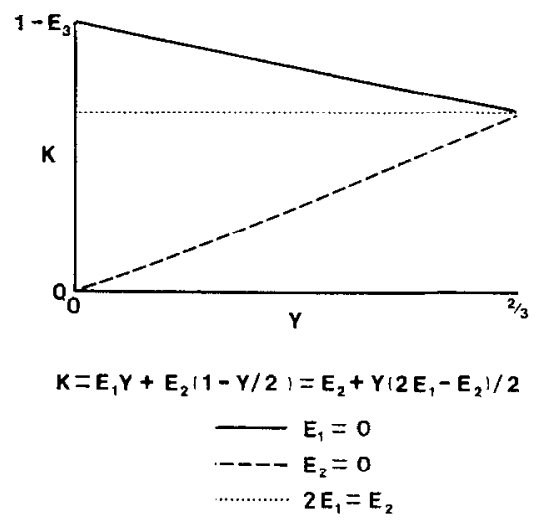

Fig. 3. A graphical representation of the dependence of $K$, the probability of a heterozygous adult giving rise to a heterozygous zygote, upon linkage to the centromere $(Y)$ and the proportions of the diploid restoring mechanisms $\left(E_{1}, E_{2}, E_{3}\right)$.

As Murdy and Carson point out, D. mangabeirai has a meiotic spindle orientation which increases the probability of central fusion and which differs from the type reported for other species of Drosophila. Therefore, they concluded that "... the system has presumably evolved under natural selection."

The other mode of P-selection is through modification of linkage relations and recombination values. This may have occured in the two natural parthenogenetic. dipterous species Drosophila mangabeirai and Lonchoptera dubia. L. dubia, like D. mangabeirai, also most likely reproduces by central fusion (Stalker, 1956). As can be seen by letting $E_{2}-1, E_{1}-E_{3}=0$ in Table I or in Fig. 3, $P$ in- 
creases for heterozygous genotypes as the recombination frequencies between the loci involved and their centromeres decreases. Hence, if the heterozygous genotypes are superior one would expect selection for factors which decrease recombination frequencies. Indeed, it is found that D. mangabeirai has many inversions which function as crossover suppressors and make whole blocks of genes effectively absolutely linked to the centromere. Thus $P$ is close to one for a large number of heterozygous genotypes, a condition Carson $(1962,1967)$ has termed "permanent heterozygosity." A similar situation exists in L. dubia (Stalker, 1956) which is characterized by inversion heterozygosity and the absence of adult structural homozygotes in wild populations. Both of these species' chromosomes and fusion mechanisms might well be an example of P-selection. However, increasing the falue of $P$ is only an advantage when heterozygotes have superior fitness, so if P-selection really is a factor in the evolution of these two species it must also be concluded that heterosis plays a very important role in maintaining the fitness of individuals of these two species.

\section{ACKNOWLEDGMENTS}

The author is very grateful to Dr. Edward D. Rothman for many helpful suggestions concerning this work and to Dr. John Vandermeer, Dr. Charles F. Sing and the referees for their critical evaluations of an earlier version of this manuscript.

\section{REFERENCES}

Asher, J. H., Jr. 1970. Parthenogenesis and genetic variability. II. One locus models for various diploid populations, Genetics 66, 369-391.

Asher, J. H., Jr. 1972. Systems of Reproduction. II. The influence of linkage and fitness upon the genetic structure of automictic parthenogenetic populations, unpublished manuscript.

Barratt, R. W., Newmeyer, D., Perkins, D. D., and Garnjobst, J. 1954. Map construction in Neurospora crassa, Advan. Genet. 6, 1-93.

Bellman, R. 1953. "Stability Theory of Differential Equations," Dover, New York.

Carson, H. L. 1962. Fixed heterozygosity in a parthenogenetic species of Drosophila, Univ. Texas Publ. 6205, 55-62.

Carson, H. L. 1967. Permanent heterozygosity, Evol. Biol. 1, 143-168.

Carson, H. L., Wei, I. Y., and Niederkorn, J. A., Jr. 1969. Isogenicity in parthenogenetic strains of Drosophila mercatorum, Genetics 63, 619-628.

Charlesworth, B. 1971. Selection in density-regulated populations, Ecology 52, 469-474.

Clarke, B. 1972. Density-dependent selection, Amer. Nat. 106, 1-13.

Gantmacker, F. R. 1959. "Matrix Theory," Vol. 2, Chelsea, New York.

GoldBerG, S. 1958. "Introduction to Differences Equations," John Wiley and Sons, New York.

KARLIN, S. 1968. Equilibrium behavior of population genetic models with non-random mating. I. Preliminary and special mating systems, J. Appl. Prob. 5, 231 313. 
Murdy, W. H., and Carson, H. L. 1959. Parthenogenesis in Drosophila mangabeirai Malog., Amer. Nat. 93, 355-363.

RoughGARDEN, J, 1971. Density-dependent natural selection, Ecology 52, 453-468.

STALKER, H. D. 1956. On the evolution of parthenogenesis in Lonchoptera (Diptera), Evol. 10, 345-359.

Templeton, A. R., AND Rothman, E. D. 1973. The population genetics of parthenogenetic strains of Drosophila mercatorum. I. One locus model and statistics, Theor. Appl. Genet. 43, 204-212.

WhITE, M. J. D. 1970. Heterozygosity and genetic polymorphism in parthenogenetic animals, in "Essays in Evolution and Genetics," (H. Hecht and W. Steere, Eds.), pp. 280-302, Appleton-Century-Croft, New York. 